\title{
PENETRATION TEST OF CAFFEINE IN ETHOSOME AND DESMOSOME GEL USING AN IN VITRO METHOD
}

\author{
ISKANDARSYAH ISKANDARSYAH*, ALVINA WIJAYA PUTERI, ERNYSAGITA ERNYSAGITA \\ Department of Pharmacy, Faculty of Pharmacy, Universitas Indonesia, Depok, Indonesia. Email: aya_2803@yahoo.com
}

Received: 21 April 2017, Revised and Accepted: 13 July 2017

ABSTRACT

Objectives: Caffeine has many functions including its use in the field of cosmetics. Nonetheless, the percutaneous absorption of caffeine is very low (9\%), and the penetration of a substance such as caffeine in the skin is not desirable. Ethosomes and desmosomes are lipid vesicles created by the modification of liposomes containing phospholipids and ethanol or dimethyl sulfoxide (DMSO) as the penetration enhancer. The purpose of this study was to compare the effectiveness of ethosomes and desmosomes as vesicles in increasing the penetration of caffeine.

Methods: Ethosomes and desmosomes were prepared using phosphatidylcholine, ethanol/DMSO, and caffeine. Phosphatidylcholine was used in the form of phospholipon $90 \mathrm{~g}$ that was obtained from soybeans. Observations were done including the characteristic of ethosomes and desmosomes, organoleptic observation, homogeneity observation, and in vitro penetration test using Franz diffusion cell method.

Results: The cumulative penetration of caffeine ethosome gel is $3316.46 \pm 218.51 \mu \mathrm{g} / \mathrm{cm}^{2}$, with flux $249.45 \pm 30.06 \mu \mathrm{g} \cdot \mathrm{cm}^{-2} \cdot \mathrm{hr}^{-1}$, and $62.35 \pm 4.52 \%$. The cumulative penetration of the desmosome gel is $2954.95 \pm 222.87 \mu \mathrm{g} / \mathrm{cm}^{2}$ with flux $381.68 \pm 34.91 \mu \mathrm{g} \cdot \mathrm{cm}^{-2} \cdot \mathrm{hr}^{-1} \mathrm{and} 53.4 \pm 3.65 \%$.

Conclusions: It can be concluded that ethosome is more effective than desmosome in increasing the penetration of caffeine.

Keywords: Caffeine, Desmosome, Ethosome, Penetration study, Phosphatidylcholine, Penetration study, Thin-layer hydration.

(C) 2017 The Authors. Published by Innovare Academic Sciences Pvt Ltd. This is an open access article under the CC BY license (http://creativecommons. org/licenses/by/4. 0/) DOI: http://dx.doi.org/10.22159/ijap.2017.v9s1.69_76

\section{INTRODUCTION}

Caffeine is an alkaloid derivate of methylxanthine and has nitrogen atoms with a two-ring or two-cycle structure [1]. Caffeine is mostly consumed in beverages and is used in medicines and cosmetics [2]. It is also used as an active substance in anti-cellulite products because it can prevent fat accumulation [3]. According to the previous study, a less amount of caffeine is absorbed (only 9\%) through the cutaneous layer [4]. In addition, the stratum corneum has a lamellar lipid layer and corneocytes that play a role in preventing the penetration of other substances, including drugs into the body [5]. It is not desirable that caffeine penetrates through the skin because it has a hydrophilic material with $\log p=-0.07^{2}$. One of the methods used to increase caffeine penetration involves making a vesicle form of caffeine. The objective of this study was to prepare caffeine ethosomes and desmosomes.

Ethosome is a modified lipid vesicle made from liposomes. We chose ethosome as the vesicle because it is suitable to be used for penetration through the skin due to its high deformability that allows it to enter the membrane. Moreover, ethosome has the advantages of low toxicity, higher permeability, the ability to encapsulate the hydrophilic and lipophilic molecules, and a smaller size than the conventional vesicles [6]. Desmosome is a newly developed lipid vesicle containing dimethyl sulfoxide (DMSO) and phospholipid. It was chosen as a vesicle because it is a new vesicle, and only a few studies have reported on it. In this study, we aimed to compare the effectiveness of ethosomes and desmosomes as vesicles to improve caffeine penetration because both vesicles contain penetration enhancers such as ethanol and DMSO, respectively. In addition, while many studies have reported about other vesicles, a few studies have researched desmosomes, particularly concerning the formation of a caffeine vesicle. Ethosome was chosen as the comparison standard because ethosomes have been widely observed and are used to improve the penetration of certain substances. Therefore, we aimed to conduct a study about the use of desmosomes and ethosomes for improving the penetration ability of caffeine. This study tested the ethosome and desmosome characteristics and compared the penetration abilities of the ethosome and desmosome preparations using the Franz diffusion cell method.

\section{MATERIALS AND METHODS}

Materials

Caffeine (Brataco, Indonesia), phospholipon 90 g (Lipoid, German), dichloromethane (Merck, German), ethanol (Merck, German), carbomer (Brataco, Indonesia), DMSO (Merck, German), TEA (Triethanolamine), and phosphate buffer (pH 7.4) (Table 1) were used.

\section{Study method}

Ethosome and desmosome preparations

Method

Phospholipon $90 \mathrm{~g}$ and caffeine were dissolved in dichloromethane, evaporated using a rotary evaporator device at $37^{\circ} \mathrm{C}$ and $50 \mathrm{rpm}$, and evaporated using vacuum; the speed of the evaporator was increased gradually every 2 minutes till it reached $150 \mathrm{rpm}$ and until the solvent completely evaporated, forming a thin layer. Nitrogen gas was passed through the thin layer that was then allowed to settle for one night; the hydration process was performed with $\mathrm{pH}$ buffer 7.45 and the ethanol or DMSO, and the thin-layer peeling was performed using glass beads.

\section{Characterization of ethosomes and desmosomes}

\section{Particle size distribution}

The particle size distribution measurement of ethosomes and desmosomes was performed using a particle size analyzer (PSA) device. A drop of the suspension was diluted in $10 \mathrm{~mL}$ distilled water; after that, the particle size was measured using the PSA device.

\section{Zeta potential}

Zeta potential is a parameter of electrical charge between colloid particles. The higher the zeta potential value, the smaller possibility of the flocculation (colloid emerging from a small particle to a big particle). The zeta potential was measured using a Zetasizer device [7]. 


\section{Morphological characterization}

Morphological characterization of the ethosomes and desmosomes was conducted using a transmission electron microscopy (TEM) device. On the copper grid (sample container), a drop of the suspension of ethosome and desmosome that had been diluted at a ratio of 1:50 was dropped and allowed to settle and be absorbed. Then, coloration was performed using uranyl acetate and dried at room temperature. After they were thoroughly dry, the ethosome morphology was analyzed using TEM.

\section{Deformability index test}

The ethosome and desmosome suspension was filtered using a polycarbonate membrane (size $0.1 \mu \mathrm{m}$ ) in a mini extruder set device. The total suspension that was filtered was determined using the following formula:

$$
D=J\left(\frac{r v}{r p}\right)^{2}
$$

$\mathrm{D}=$ deformability index

$\mathrm{J}=$ total ethosome or desmosome suspension that passed through the membrane $(\mathrm{mL})$

$\mathrm{rv}=$ particle size of the ethosome or desmosome that passed through the membrane (nm)

$\mathrm{rp}=$ membrane pore size $(\mathrm{nm})$.

\section{Formulation of the ethosome and desmosome gel}

The formulation of the ethosome and desmosome gel is shown in Table 2 .

\section{Gel preparations}

Carbomer was dispersed into the distilled water overnight until the carbomer was thoroughly soaked; thereafter, it was homogenized using a homogenizer at a speed of $300 \mathrm{rpm}$, which was increased gradually. The TEA and propylene glycol were added, and the mixture was homogenized again. The ethosome and desmosome suspensions were added to the gel base. The gel was homogenized using a homogenizer at a speed of $300 \mathrm{rpm}$, which was gradually increased, until it was mixed well.

\section{Evaluation gel}

\section{Organoleptic observation}

The gel was observed to identify any possible incidents, including the formation of a separate phase (syneresis), development of an odor, or a change in the color.

Table 1: Formulation of ethosome and desmosome

\begin{tabular}{lll}
\hline Ingredients & Concentration & Function \\
\hline Caffeine & $500 \mathrm{mg}$ & Active substance \\
Phospholipon $90 \mathrm{~g}$ & $4 \mathrm{~g}$ & Vesicle \\
DMSO/ethanol & $5 \mathrm{~mL}$ & Penetration enhancer \\
Buffer pH 7.5 & $\mathrm{Ad} 25 \mathrm{~mL}$ & Solvent \\
\hline
\end{tabular}

DMSO: Dimethyl sulfoxide

Table 2: Formulation of the ethosome and desmosome gel

\begin{tabular}{llll}
\hline Materials & \multicolumn{2}{l}{ Concentration\% (b/b) } & \\
\cline { 2 - 4 } & $\begin{array}{l}\text { Ethosome } \\
\text { gel }\end{array}$ & $\begin{array}{l}\text { Desmosome } \\
\text { gel }\end{array}$ & Function \\
\hline $\begin{array}{l}\text { Caffeine } \\
\text { ethosome }\end{array}$ & 1.5 & - & Active substance \\
$\begin{array}{l}\text { Caffeine } \\
\text { desmosome }\end{array}$ & - & 1.5 & Active substance \\
$\begin{array}{l}\text { Carbomer } \\
\begin{array}{l}\text { Propylene glycol } \\
\text { TEA }\end{array}\end{array}$ & 0.5 & 0.5 & $\begin{array}{l}\text { Gelling agent } \\
\text { Aqua }\end{array}$ \\
$\begin{array}{l}\text { demineralisata } \\
\text { demectant }\end{array}$ \\
\hline
\end{tabular}

\section{Homogeneity observation}

The gel was placed between two glass objects and observed under the light. Any observed irregularities or coarse particles were recorded. This observation was conducted for 8 weeks, during which time, the gel was observed every 2 weeks.

\section{In vitro penetration test using the Franz diffusion cell method}

This test was performed over a period of $8 \mathrm{hrs}$, and the samples were collected at the following 11-time points: 10, 30, 60, 90, 120, 180, 240, $300,360,420$, and 480 minutes. The volume of each collected sample was $1 \mathrm{~mL}$ to which $1 \mathrm{~mL}$ buffer ( $\mathrm{pH} 7.4)$ was added. The total amount of caffeine that penetrated, per diffusion area $\left(\mu \mathrm{g} / \mathrm{cm}^{2}\right)$, was measured using the following formula:

$$
Q=\frac{\left[C n . V+\sum_{i=1}^{n-1} C . S\right]}{A}
$$

$\mathrm{Q}=$ Total caffeine that penetrated per diffusion area $\left(\mu \mathrm{g} / \mathrm{cm}^{2}\right)$

$\mathrm{Cn}=$ concentration of caffeine $(\mu \mathrm{g} / \mathrm{mL})$

$\mathrm{V}=$ volume of the Franz diffusion cell

$\sum_{i=1}^{n-1}=$ concentration of caffeine $(\mu \mathrm{g} / \mu \mathrm{L})$ in the first sample (collected at 10 minutes)

$\mathrm{S}=$ volume of the samples $(1000 \mu \mathrm{L})$

$\mathrm{A}=$ membrane area $\left(2.54 \mathrm{~cm}^{2}\right)$

\section{RESULTS AND DISCUSSIONS}

Ethosome and desmosome preparation

Ethosomes and desmosomes were prepared using phosphatidylcholine, ethanol/DMSO, and caffeine. Phosphatidylcholine was the main component for vesicle formation because it has polar and nonpolar groups that form a lipid bilayer structure in the vesicle. Phosphatidylcholine was used in the form of phospholipon $90 \mathrm{~g}$ that was obtained from soybeans. Phospholipon $90 \mathrm{~g}$ was chosen because the ethosome suspension formulation would be applied as a topical preparation, and the phosphatidylcholine from the soybeans would probably not have a bad smell like the phosphatidylcholine obtained from eggs. Ethanol and DMSO were chosen as the penetration enhancers of the hydrophilic active substance caffeine. The thin-layer hydration method was chosen because it is a simple method that can be easily performed. This method included the two steps of layer formation and hydration. The ethosome and desmosome suspension obtained was cloudy white, thick, and had a typical odor. This may have been because caffeine, the active substance, had a white color, while the specific odor came from phosphatidylcholine, which is known to have a typical odor.

\section{Ethosome and desmosome evaluation}

\section{Particle size distribution and zeta potential}

The mean sizes of the ethosomes and desmosomes were $106.6 \mathrm{~nm}$ and $388.4 \mathrm{~nm}$, respectively. The sizes of both the vesicles $(<1000 \mathrm{~nm})$ were considered appropriate for nanovesicle preparation. Ethosomes were smaller than the desmosomes because the molecule weight of ethanol is $46.07 \mathrm{~g} / \mathrm{mol}$ while that of the DMSO molecule is $78.133 \mathrm{~g} / \mathrm{mol}$. The zeta potentials, analyzed using a Zetasizer, of the ethosomes and desmosomes were $-27.8 \mathrm{mv}$ and $-37.2 \mathrm{mv}$, respectively. This indicates that the desmosome suspension was more stable than the ethosome suspension, and the zeta potential value of the desmosome suspension met the standard requirements of the zeta potential suspension parameter $( \pm 30 \mathrm{mv})$. The polydispersity index values of the ethosome and desmosome suspensions were 0.313 and 0.823 , respectively. This showed that the ethosomes had a more homogenous suspension than the desmosomes.

\section{Morphological characterization}

According to the morphological analyses of the ethosomes and desmosomes conducted using a TEM device (Fig. 1), we found that the 
caffeine attached on the phosphatidylcholine head which was polar marked with a black circle layered with a white circle. The vesicle obtained was identified as a large unilamellar vesicle based on the fact that there was only one lamella in the vesicle with particle size 100$1000 \mathrm{~nm}$.

\section{Deformability index}

The deformability index is a parameter used to compare the flexibility of the ethosome and desmosome preparations. The higher the deformability index, the more flexible the vesicle. Desmosome had a higher deformability index than ethosome (15.08 vs. 1.13). This may be attributable to the fact that the particle size of the desmosome was larger than that of the ethosome (388.4 nm vs. $106.6 \mathrm{~nm}$ ). Therefore, when passing through a 100-nm membrane, desmosomes would have a higher deformability index than ethosomes.

\section{Ethosome and desmosome caffeine gel preparation}

The gel form was chosen because according to the previous study, gels provide better penetration than other semi-solid preparations such as cream and ointments. Ethosome and desmosome caffeine gels were prepared using carbomer. Carbomer provided a better viscosity in gel preparations and was added to create a gel base with a moderate viscosity to encourage the dispersion process when applied to the skin. Distilled water was used to eliminate the mineral content that would decrease the $\mathrm{pH}$ value, making it more acidic. A concentration of $1.5 \%$ of the active substance was used for preparing the ethosome and desmosome suspensions to be used for making the gels. This was based on a previous study that used $1-2 \%$ caffeine to produce an anti-cellulite effect. The gel base was prepared at a low speed of $300 \mathrm{rpm}$ to prevent trapping of air in the gel. This was done to ensure that the gel produced would be homogenous and without bubbles and that there would be no damage to the ethosomes and desmosomes.

\section{Gel evaluation}

The organoleptic observation was employed for gel evaluation. The gel formulations of ethosome and desmosome were not clear (in color). Both the gels were white and had a typical odor. The gel was white because the ethosome and desmosome suspensions were white. The specific odor of the gel was because both the suspensions had a characteristic odor due to the phospholipid used. The gels did not exhibit syneresis or phase separation. The results showed that the ethosome gel preparation had a $\mathrm{pH}$ of 5.19, whereas the desmosome gel had a pH of 5.47. The $\mathrm{pH}$ of the gel should ideally be within the range of the $\mathrm{pH}$ skin, i.e. between 4.5 and 6.5 . If the $\mathrm{pH}$ is too low (acidic), the gel would irritate the skin; however, if it is too high (alkaline), the skin would become scaly. The ethosome gel had a viscosity value of $9600 \mathrm{cps}$, whereas the desmosome gel had a viscosity value of 9200 cps. The desmosome gel had a higher viscosity than the ethosome gel. This could be because the desmosome suspension was thicker than the ethosome suspension. Neither of the gel formulations underwent deformation from the original gel preparation before the cycling test, and they did not exhibit syneresis or phase separation, indicating that both the gel preparations were stable in the cycling test.

\section{In vitro penetration test using the Franz diffusion cell method}

This penetration test was conducted to define the amount of caffeine from the gels that could penetrate the skin in a certain period. This test was performed using membrane as skin model to maintain the low concentration or usually called sink condition. After the sample collection at the 11-time points, we found that the caffeine absorption rate from the ethosome gel was the highest at $8 \mathrm{hrs}$, and the total accumulated penetration was $3.317 .53 \pm 235.28 \mu \mathrm{g} / \mathrm{cm}^{2}$. In contrast, the desmosome gel showed a total accumulated penetration as $2.958 .10 \pm 190.52 \mu \mathrm{g} / \mathrm{cm}^{2}$. This showed that the total accumulated penetration was higher for the ethosome gel than for the desmosome gel (Fig. 2). In the ethosome gel, the flux obtained during 4-8 hrs was expected to be the steady state of the ethosome gel. In the desmosome gel, the flux obtained in during 1-8 hrs was expected to be the steady state of the desmosome gel. The flux of the ethosome gel was $249.45 \pm 30.06 \mu \mathrm{g} \cdot \mathrm{cm}^{-2} \cdot \mathrm{hr}^{-1}$, whereas it was $381.68 \pm 34.91 \mu \mathrm{g} \cdot \mathrm{cm}^{-2} \cdot \mathrm{hr}^{-1}$ for the desmosome gel (Fig. 3). These results showed that the desmosome gel had a higher flux than the ethosome gel (Fig. 4), indicating that the caffeine penetration rate in the desmosomes was more constant than that in the ethosome gel although the total accumulated penetration was higher in the ethosome gel. The percentage of the total penetrated caffeine of the ethosome gel was $62.39 \pm 4.52 \%$, whereas it was $53.44 \pm 3.65 \%$ for the desmosome gel. The

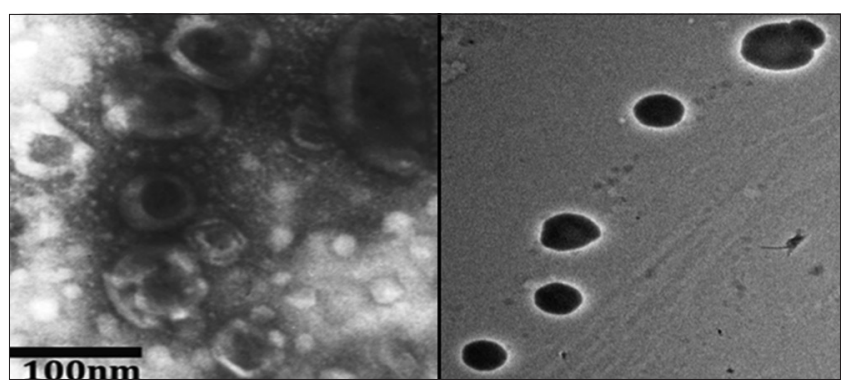

Fig. 1: Ethosome morphology (left) and desmosome morphology (right)

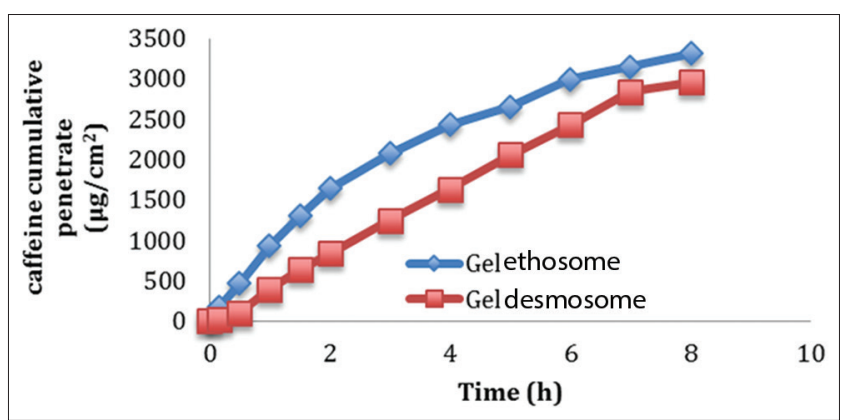

Fig. 2: Total cumulative caffeine penetration by the ethosome and desmosome gels

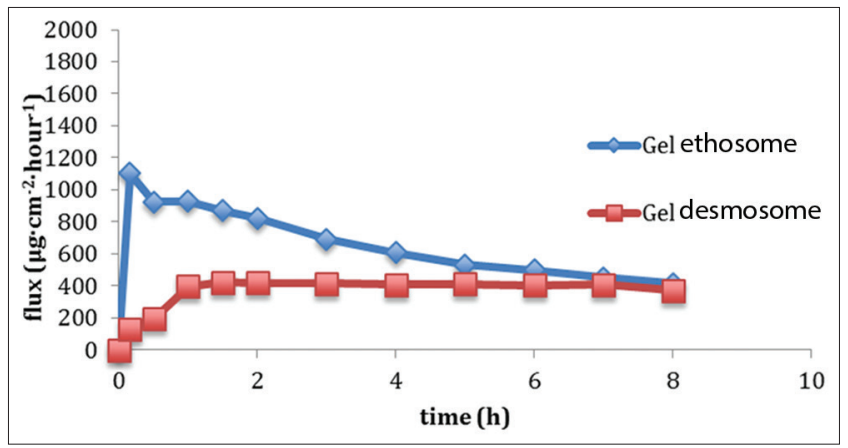

Fig. 3: Flux (per time) of the ethosome and desmosome gels

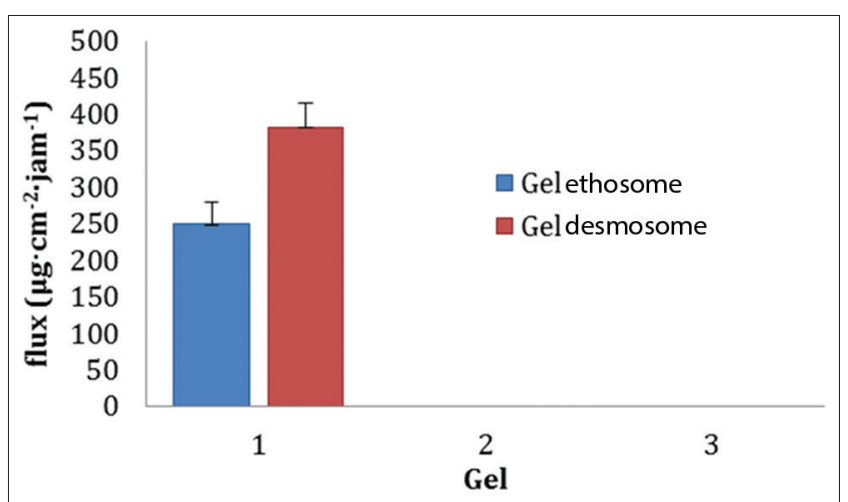

Fig. 4: Flux of caffeine from the ethosome and desmosome gels 
percentage of the total penetrated caffeine was higher in the ethosome gel than in the desmosome gel; however, these percentages were not significantly different because both the gels were in the vesicle form and both used penetration enhancers. Another reason for this difference in the percentages was the smaller particle size of the ethosomes, making it easy for the ethosomes to penetrate through the membrane even with ethanol addition and DMSO obtained in lipid vesicle worked as the penetration enhancer. Ethanol works cutaneously as a penetration enhancer by changing the dilution property of the stratum corneum, resulting in the greater dissolution of caffeine in the stratum corneum, leading to increased penetration [8]. On the other hand, the DMSO works as a penetration enhancer by causing the denaturation of the skin proteins, which experience keratin conformation intercellular from $\alpha$-helix conformation into $\beta$-sheet conformation [9].

\section{Skin}

The skin is the outermost part of the body. The skin surface area in an adult is approximately $2 \mathrm{~m}^{2}$, and the skin weight is about $15 \%$ of the total body weight [10]. Skin is an essential and vital organ and is a marker of health. It is very complex, elastic, and sensitive, and varies according to the temperature, age, sex, race, and body location [11].

\section{Caffeine}

Caffeine is a heterocyclic alkaloid of the methyl xanthine group, containing nitrogen atoms with two rings or two cyclic structures. This substance is a secondary metabolite obtained from plants found in more than 60 plants, including camellia sinensis (1-4.8\%), coffea arabica (1-1.5\%), and theobroma cacao (2.7-3.6\%). Caffeine can be consumed in beverages or used in medicines and cosmetic products [2]. It is widely used as an active substance in creams and lotions because it is believed to delay skin aging, absorb ultraviolet radiation, and prevent tumor formation in sunexposed skin. Caffeine is also used as an active substance in anti-cellulite products because it can prevent fat accumulation.

\section{Ethosome}

Ethosome is an ultra-deformable lipid vesicle obtained by modifying liposomes, developed by Williams and Barry in 2007. Ethosomes consist of phospholipid and alcohol in high concentrations (20-45\%) [12] and can increase the skin penetration of the active substance because of the ethanol interdigitation effect on the lipid bilayer and by increasing the lipid fluidity of the stratum corneum. Ethosomes may have various sizes, ranging from $10 \mathrm{~nm}$ to $\mu$ size. In addition, ethosomes have the ability to entrap active substances that are formed with a smaller size, making membrane permeation possible.

\section{Desmosome}

Desmosome is a newly developed lipid vesicle containing DMSO and phospholipid. The mechanism of desmosome differs from that of transfersome; desmosome functions as a penetration enhancer, while the transfersome improves vesicle deformability [13]. DMSO works as a skin penetration enhancer, improving skin permeability by the diffusion process, wherein the keratin conformation of the stratum corneum changes from $\alpha$-helix conformation to $\beta$-sheet conformation [9].

\section{CONCLUSION}

Based on the results of the characterization and penetration tests, it can be concluded that the ethosome gel is more effective than the desmosome gel. Future research that uses in vivo assays to determine the usefulness of the caffeine-containing ethosome and desmosome gels in the field of cosmetics is warranted, considering that the desmosome vesicle is relatively new and has not been well researched. Furthermore, ethosome and desmosome particles of the same size should be produced to determine which vesicle enables deeper penetration.

\section{REFERENCES}

1. Misra H, Mehta D, Mehta BK, Soni M, Jain DC. Study of extraction and HPTLC - UV method for estimation of caffeine in marketed tea (Camellia sinensis) granules. Int J Green Pharm 2009;3:47-51.

2. Luo L, Lane ME. Topical and transdermal delivery of caffeine. Int $\mathrm{J}$ Pharm 2015;490(1-2):155-64.

3. Gajewska M, Paini A, Sala Benito JV, Burton J, Worth A, Urani C, et al. In vitro-to-in vivo correlation of the skin penetration, liver clearance and hepatotoxicity of caffeine. Food Chem Toxicol 2015;75:39-49.

4. Walters KA. Dermatological and Transdermal Formulations. New York: Marcel Dekker; 2002.

5. Djajadisastra J. Cosmetic Stability. Presented in HIKI Half Day Conference. Jakarta, $18^{\text {th }}$ November; 2004

6. Razavi H, Janfaza S. Ethosome: A nanocarrier for transdermal drug delivery. J Paramed Sci 2015;6(2):2-7.

7. Malika V, Kohli K, Chaudhary H, Kumar V. Nano-carrier for accentuated transdermal drug delivery. J Dev Drugs 2014;3(2):1-9.

8. Williams AC, Barry BW. Chemical permeation enhancement. In: Touitou E, Barry BB, editors. Enhancement in Drug Delivery. United States of America: CRC Press; 2007.

9. Trommer H, Neubert RH. Overcoming the stratum corneum: The modulation of skin penetration. A review. Skin Pharmacol Physiol 2006;19(2):106-21.

10. Alexander A, Dwivedi S, Ajazuddin, Giri TK, Saraf S, Saraf S, et al. Approaches for breaking the barriers of drug permeation through transdermal drug delivery. J Control Release 2012;164(1):26-40.

11. Wasiaatmadja SM. Cosmetical Medic. Jakarta: UI-Press; 1997.

12. Rakesh R, Anoop KR. Ethosomes for transdermal and topical drug delivery. Int J Pharm Pharm Sci 2012;4 Suppl 3:17-24.

13. Menezes AC. Management of Skin Cancer by Agonist of 5-HT1A and Antagonist of 5-HT2A. Thesis. Lisboa, Portugal: Universidade de Lisboa; 2015. 